\title{
Low-altitude frequency-banded equatorial emissions observed below the electron cyclotron frequency
}

\author{
Mohammed Y. Boudjada ${ }^{1}$, Patrick H. M. Galopeau ${ }^{2}$, Sami Sawas ${ }^{3}$, Valery Denisenko ${ }^{4,5}$, Konrad Schwingenschuh $^{1}$, \\ Helmut Lammer ${ }^{1}$, Hans U. Eichelberger ${ }^{1}$, Werner Magnes ${ }^{1}$, and Bruno Besser ${ }^{1}$ \\ ${ }^{1}$ Space Research Institute, Austrian Academy of Sciences, Graz, Austria \\ ${ }^{2}$ LATMOS-CNRS, Université Versailles Saint-Quentin-en-Yvelines, Guyancourt, France \\ ${ }^{3}$ Institute of Communications and Wave Propagation, Graz University of Technology, Graz, Austria \\ ${ }^{4}$ Institute of Computational Modelling, Russian Academy of Sciences, Krasnoyarsk, Russia \\ ${ }^{5}$ Siberian Federal University, Krasnoyarsk, Russia
}

Correspondence: Mohammed Y. Boudjada (mohammed.boudjada@oeaw.ac.at)

Received: 22 March 2019 - Discussion started: 3 April 2019

Revised: 6 May 2020 - Accepted: 8 May 2020 - Published: 24 June 2020

\begin{abstract}
The ICE (Instrument Champ Électrique) experiment on board the DEMETER (Detection of ElectroMagnetic Emissions Transmitted from Earthquake Regions) satellite recorded frequency-banded wave emissions below the electron cyclotron frequency, with band spacing $\gtrsim$ frequency low-hybrid resonance, in the vicinity of the magnetic equatorial plane. Those radiations were observed in the beginning of the year 2010 on the night side of Earth and rarely on the day side. We distinguish two components: one appears as frequency bands continuous in time between a few kilohertz and up to $50 \mathrm{kHz}$, and the other one is from 50 to $800 \mathrm{kHz}$. The first component exhibits positive and negative frequency drift rates in the Southern Hemisphere and Northern Hemisphere, at latitudes between 40 and $20^{\circ}$. The second one displays multiple spaced frequency bands. Such bands mainly occur near the magnetic equatorial plane with a particular enhancement of the power level when the satellite latitude is close to the magnetic equatorial plane. We show in this study the similarities and the discrepancies between the non-free-space DEMETER frequency-banded emissions and the well-known free-space terrestrial kilometric radiation. The hollow cones of the DEMETER frequency-banded wave emissions are oriented towards Earth's ionosphere. We suggest that the source region is localized in regions poleward of the plasmapause where the ratio of the plasma frequency to gyro-frequency is smaller than one.
\end{abstract}

\section{Introduction}

A variety of radio waves were detected in the near-Earth space environment in the 1970s. The first type of waves were observed at frequencies below 100 and up to $30 \mathrm{kHz}$ (Brown, 1973) and even lower between 5 and $20 \mathrm{kHz}$ (Gurnett and Shaw, 1973). These two types of emission belong to a single non-thermal continuum spectrum, one "trapped" and the other "escaping" (Gurnett, 1975). High-resolution spectrograms also made evident the presence of numerous narrow-band emissions for the escaping component (Kurth et al., 1981). Later on, the Cluster tetrahedral configuration of four identical satellites allowed for the analysis of a specific type of a nonthermal continuum (Décréau et al., 2001). A direction-finding technique, based on antenna spin modulation, allowed for localizing the source regions in the plasmapause (Décréau et al., 2004), confirming previous Geotail observations (Hashimoto et al., 1999). Grimald et al. (2008) showed in the nonthermal emissions the presence of spectral peaks organized as several banded emissions with a frequency interval nearby the gyrofrequency at the sources. The considered event was recorded on 30 December 2003, in the Southern Hemisphere and Northern Hemisphere before and after plasmapause boundaries. All satellites display a similar behaviour with arranged peaking times (i.e. C1, C2, C3 and then $\mathrm{C} 4$ ) corresponding to the satellite ordering along the "line of pearls configuration". Authors suggested a stable beam of a limited cone angle. Further polarization investigations of such a type of banded emissions by Grimald and 
Santolik (2010) led to the conclusion that the observed polarization excludes the presence of the Langmuir mode and the ordinary mode. Details on the wave spectral signature were also investigated by El-Lemdani Mazouz et al. (2009), particularly the splitting in fine frequency bands. Another type called "nonthermal continuum patches" was found to occur within a relatively short time and over a wide frequency range (Grimald et al., 2011). Authors showed that plasmaspheric "patch" events represent $25 \%$ of the total nonthermal emissions recorded in 1 year.

Also space observations provided by the IMAGE (Imager for Magnetopause-to-Aurora Global Exploration) satellite (Burch, 2000) allowed for a better investigation of the inner plasmasphere. The Radio Plasma Imager (RPI) was designed to use a radio sounding technique leading the reception of echoes from remote plasma regions. Emitted pulses can propagate in the $Z$ mode and also the whistler mode (Carpenter et al., 2003). Hence signals detected at frequencies below the local upper-hybrid frequency $f_{\mathrm{uh}}=\left(f_{\mathrm{p}}^{2}+f_{\mathrm{g}}^{2}\right)^{1 / 2}$ could propagate in the whistler and $Z$ modes. Here $f_{\mathrm{p}}$ and $f_{\mathrm{g}}$ are the plasma frequency and the gyro-frequency, respectively. Sonwalkar et al. (2004) showed that the $f_{\mathrm{p}} / f_{\mathrm{g}}$ ratio leads to the sounding of different regions of the plasmasphere. Hence the condition $f_{\mathrm{p}} / f_{\mathrm{g}}>1$ allows for sounding below 2000 and above $4000 \mathrm{~km}$ within Earth's plasmasphere. In regions poleward of the plasmapause, the second condition prevails, i.e. $f_{\mathrm{p}} / f_{\mathrm{g}}<1$. Similar plasma conditions were derived from the theoretical approach by Goertz and Strangeway (1995) using the Appleton-Hartree dispersion relation.

In this paper, we analyse the frequency-banded radiation observed by the ICE (Instrument Champ Électrique) DEMETER (Detection of Electro-Magnetic Emissions Transmitted from Earthquake Regions) experiment in the beginning of the year 2010. The characteristics of this radiation, essentially the spectral features and the spatial occurrence, are described in Sect. 2. A discussion of the outcomes is detailed in Sect. 3, where principally our results are combined with previous ones. A summary of the main results is given in Sect. 4 .

\section{Frequency-banded wave emission}

\subsection{Overview of high-frequency ICE observations}

We consider in this study the space observations provided by the DEMETER microsatellite. The aim is the analysis of particular spectral features recorded by the ICE experiment in the beginning of the year 2010, i.e. January, February and March. The ICE instrument allows for a continuous survey of the electric field over a wide frequency range, from a few hertz up to about $3.5 \mathrm{MHz}$ (Berthelier et al., 2006). The electric field component is determined along the axis defined by two sensors. The satellite sun-synchronous half-orbit duration is about $40 \mathrm{~min}$, covering the invariant latitude between -65 and $+65^{\circ}$. The DEMETER satellite orbits are associ- ated with two fixed local times, at about 10 and 22 LT. We use in this investigation the survey mode of the ICE experiment covering the frequency range between a few kilohertz and 3.5 MHz, called hereafter the HF (high-frequency) band. The radio wave emissions are alternately recorded on the day and night sides of Earth corresponding respectively to down and up half orbits. However the main radiations investigated in this paper are observed on the night side and rarely on the day side. Generally the ICE HF-band dynamic spectra allow for distinguishing three kinds of spectral emissions depending on the satellite geographical latitudes. The first one is recorded close to the sub-auroral regions at latitudes between 50 and $60^{\circ}$; it mainly concerns the auroral kilometric radiation described by Parrot and Berthelier (2012). The second are mainly ground-based transmitters, low-frequency (LF) radiation, appearing at mid latitudes between 50 and $20^{\circ}$, in both hemispheres (e.g. Parrot et al., 2009; Boudjada et al., 2017).

The third kind of emission is a frequency-banded wave radiation occurring in the vicinity of the equatorial magnetic plane at low latitudes. Hereafter we focus on the analysis of the banded radiation, in particular the spectral characteristics, the magnetic latitude and the power intensity occurrence. Also the dependence of the power level on the frequency and the magnetic latitude is considered. We use a manual technique which consists of following and saving the frequency and the temporal evolution of the radiation with a computer mouse. The saved parameters are the observation time (UT), the frequency $(\mathrm{kHz})$ and the power level $\left(\mu \mathrm{V} \mathrm{m}^{-1} \mathrm{~Hz}^{-1 / 2}\right)$. The collected points are later combined with the satellite orbital parameters like the magnetic latitude and the $L$ shell.

\subsection{Frequency and time characteristics}

The DEMETER ICE experiment detected frequency-banded emissions in the frequency range between a few kilohertz and up to $800 \mathrm{kHz}$. Two examples recorded on the night side are shown in Figs. 1 and 2. Figure 1a displays the dynamic spectrum recorded by the ICE experiment on 21 February 2010 between 13:52 and 14:12 UT. The satellite was on the lateevening sector, around $22 \mathrm{LT}$, at a distance of $665 \mathrm{~km}$. In this time interval the satellite geographical coordinate varied from $-18^{\circ} \mathrm{S}$ to $+04^{\circ} \mathrm{N}$ in latitude and 142 to $138^{\circ}$ in longitude. The second event shown in the first panel of Fig. 2 was also recorded at about $22 \mathrm{LT}$ at a similar distance from Earth. Satellite geographical coordinates varied from $-25^{\circ} \mathrm{S}$ to $40^{\circ} \mathrm{N}$ in latitude and 117 to $102^{\circ}$ in longitude in the time interval between 14:10 and 14:26 UT. Figures $1 \mathrm{~b}$ and $2 \mathrm{~b}$ display a zoomed-in part of the dynamic spectrum shown in the first panels where the emission appears in the frequency range between a few kilohertz and up to $800 \mathrm{kHz}$. Note for Figs. $1 \mathrm{~b}$ and $2 \mathrm{~b}$ the changes in the spectral emissions before and after $50 \mathrm{kHz}$. Hence the first radiation appears as a narrow continuum with an instantaneous bandwidth of about $2 \mathrm{kHz}$ at frequencies less than $50 \mathrm{kHz}$. It displays negative 
(a)

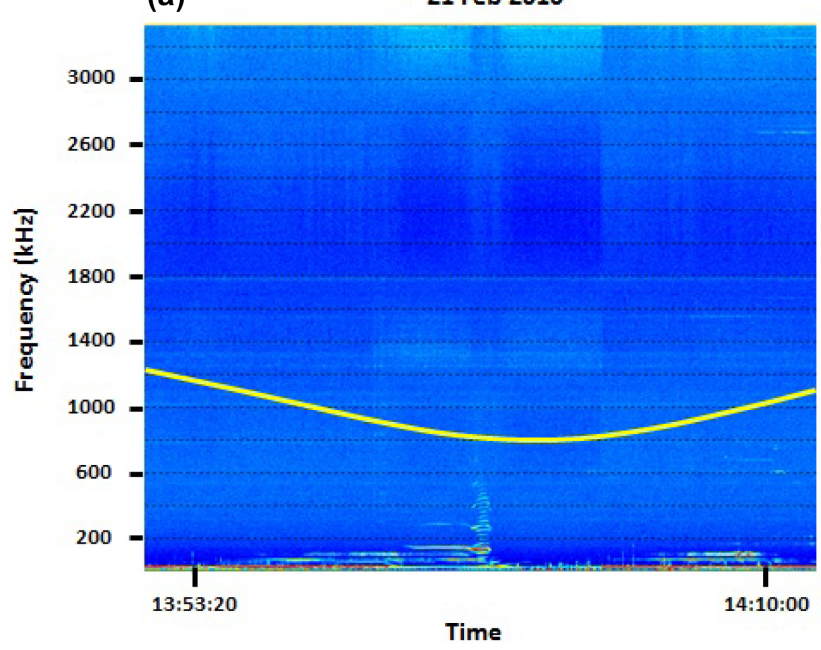

(b) 21 Feb 2010

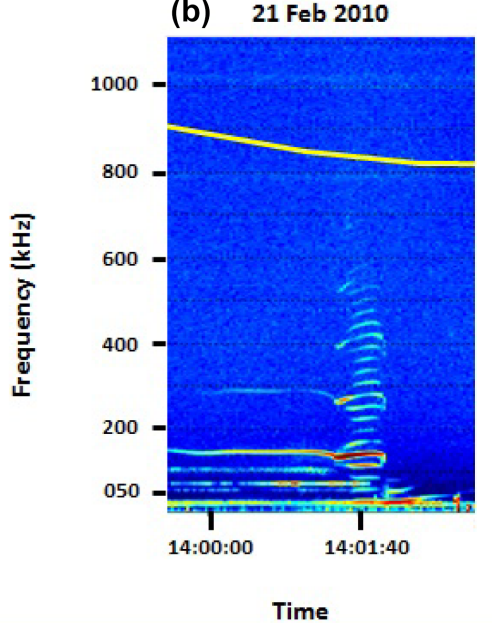

Figure 1. Example of frequency-banded wave emission recorded by the ICE experiment on board DEMETER on 21 February 2010. Panel (a) displays an overview of the dynamic spectrum in the frequency range from a few kilohertz to $3.5 \mathrm{MHz}$. Panel (b) shows a zoomed-in part for the event in the frequency bandwidth between a few kilohertz and $1100 \mathrm{kHz}$. The gyro-frequency is indicated by the yellow curve.

and positive frequency drifts when the satellite is approaching or leaving the equatorial plane, respectively. Its frequency drift rate is weak and in the order of $0.2 \mathrm{kHz} \mathrm{s}^{-1}$. The second emission is composed of parallel narrow bands for frequencies above 50 and up to $800 \mathrm{kHz}$. The band time duration is, on average, about $1 \mathrm{~min}$ and decreases to less than $1 \mathrm{~min}$ when the emission frequency increases.

The number of parallel narrow bands is found to be different from one event to another. Hence we find, respectively, 18 and 20 parallel bands on 21 February 2010 (Fig. 1) and 13 March 2010 (Fig. 2). The spacing of the frequency band, on average, is about $30 \mathrm{kHz}$ when we consider both events.
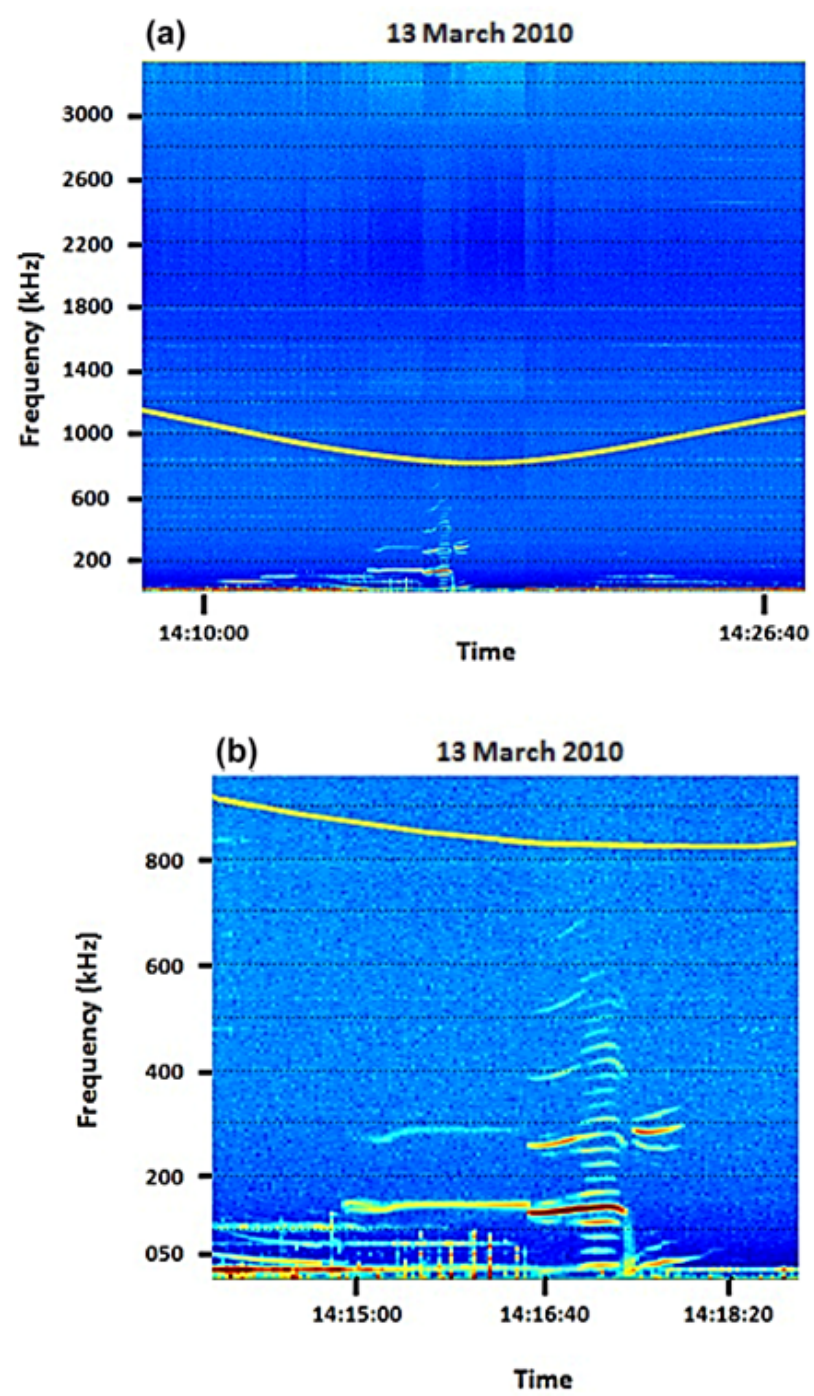

Figure 2. Like in Fig. 1 for an event recorded by DEMETER on 13 March 2010.

One can note that some narrow bands showed a high power level (red in Figs. 1 and 2), when they are compared to other narrow bands, and they also exhibit an extensive time duration of about a few minutes. Such enhanced narrow bands appear at 140, 270 and $540 \mathrm{kHz}$ in Fig. 1 and at 130, 250, 410 and $550 \mathrm{kHz}$ in Fig. 2. The enhanced banded frequencies above $200 \mathrm{kHz}$, may be considered harmonic components of a "fundamental" frequency which appears around $140 \mathrm{kHz}$ and which exhibits a longer time duration. It follows that five short weak narrow-band emissions separate the basic frequency, i.e. $140 \mathrm{kHz}$, from its first harmonic around $280 \mathrm{kHz}$. Hereafter, we consider a statistical analysis of all events observed in January, February and March 2010. 

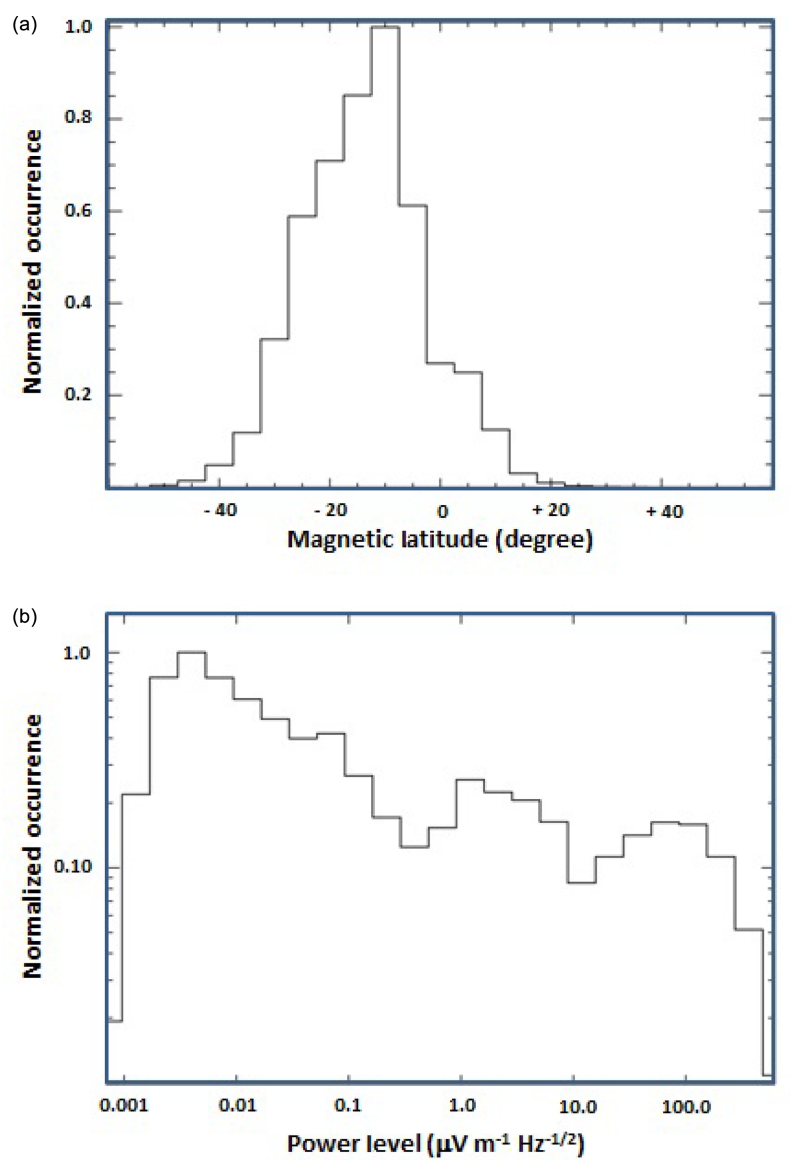

Figure 3. Occurrence of frequency-banded radiations in (a) magnetic latitude (degrees) and (b) power level $\left(\mu \mathrm{V} \mathrm{m}^{-1} \mathrm{~Hz}^{-1 / 2}\right)$.

\subsection{Magnetic latitude and power level occurrence}

The frequency-banded radiation occurrences in magnetic latitude and power level are shown, respectively, in Fig. 3a and $b$. The main emissions were recorded when DEMETER was in the southern part of the magnetic equatorial plane. Hence the emissions are detected in the magnetic latitude range between -40 and $20^{\circ}$, as shown in Fig. 3a. We note a clear progressive increase of the frequency-banded emission occurrence which reaches a maximum at a magnetic latitude of $-10^{\circ}$. More than $90 \%$ of the radiation occurred at a range in magnetic latitude between -30 and $0^{\circ}$. A sudden decrease of the occurrence is recorded when the satellite crosses the magnetic equatorial plane. Emission is found to be more extended in the Southern Hemisphere with a clear di-symmetry occurrence before and after the equatorial magnetic plane.

The power level, as displayed in Fig. 3b, covers a large interval between $10^{-3}$ and $10^{+4} \mu \mathrm{V} \mathrm{m}^{-1} \mathrm{~Hz}^{-1 / 2}$. More than $70 \%$ of emissions have a level less than $1 \mu \mathrm{V} \mathrm{m}^{-1} \mathrm{~Hz}^{-1 / 2}$ and belong mainly to the Southern Hemisphere. Above this weak power level, the occurrence of the frequency-banded emission is associated with both hemispheres. The intense

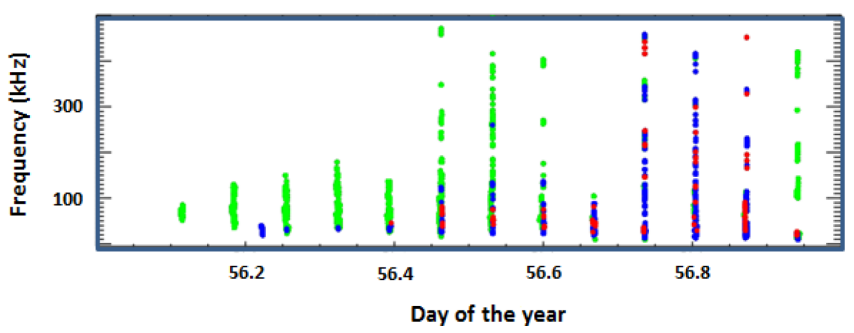

Figure 4. Vertical lines indicate the occurrence of the frequencybanded emissions observed on 25 February 2010. Those events were recorded on the night side of Earth with a time interval of about $1 \mathrm{~h} 35 \mathrm{~min}$. Green, blue and red specify, respectively, three power level intervals, i.e. $10^{-3}-0.7,0.7-10$ and $10-$ $10^{+4} \mu \mathrm{V} \mathrm{m}^{-1} \mathrm{~Hz}^{-1 / 2}$.

power level is associated with the emission occurring mainly at lower frequency, i.e. from a few kilohertz and up to $100 \mathrm{kHz}$. We distinguish three occurrence maxima at about $5 \times 10^{-3}, 1$ and $80 \mu \mathrm{V} \mathrm{m}^{-1} \mathrm{~Hz}^{-1 / 2}$. We separate the power level by taking into consideration the interval associated with previous maxima. Hereafter green, blue and red indicate, respectively, three power level intervals, i.e. 0.001-0.7, 0.7-10 and $10-10^{4} \mu \mathrm{V} \mathrm{m}^{-1} \mathrm{~Hz}^{-1 / 2}$.

Frequency-banded wave emissions are regularly observed on the night side (22 LT) before and after the magnetic equatorial plane in the vicinity of Earth at a distance less than $750 \mathrm{~km}$. Figure 4 displays the daily occurrence of frequencybanded emissions on 25 February 2010. We observe a periodic occurrence of the emission with a time interval of about $1 \mathrm{~h} 35 \mathrm{~min}$ which corresponds to a full orbit of the DEMETER microsatellite. Each vertical line is considered an "event" and corresponds to the recorded emission for a given half orbit. The occurrence per day is about 13 events in the optimal case. However from one event to another we find a variation in the frequency bandwidth and also in the power level.

\subsection{Power level versus frequency and magnetic latitude}

Figure 5 displays the power level variation versus the magnetic latitude where the colours indicate different power levels as defined in the previous subsection. The weakest intensities (less than $0.7 \mu \mathrm{V} \mathrm{m}^{-1} \mathrm{~Hz}^{-1 / 2}$ ) are recorded at magnetic latitudes between -50 and $+30^{\circ}$ but much more in the Southern Hemisphere, as displayed in Fig. 5a. Structured emissions appear when the magnetic latitude is positive principally after the crossing of the magnetic equatorial plane. One can distinguish five components appearing in four frequency ranges: a few kilohertz to 50,70 to 130 , 170 to 250,280 to 340 and 380 to $420 \mathrm{kHz}$. Those radiations are extended in magnetic latitudes in particular at low frequencies around $50 \mathrm{kHz}$ and decrease at higher frequencies at about $400 \mathrm{kHz}$. Frequency-banded emission is quasiabsent between those four frequency bands. 

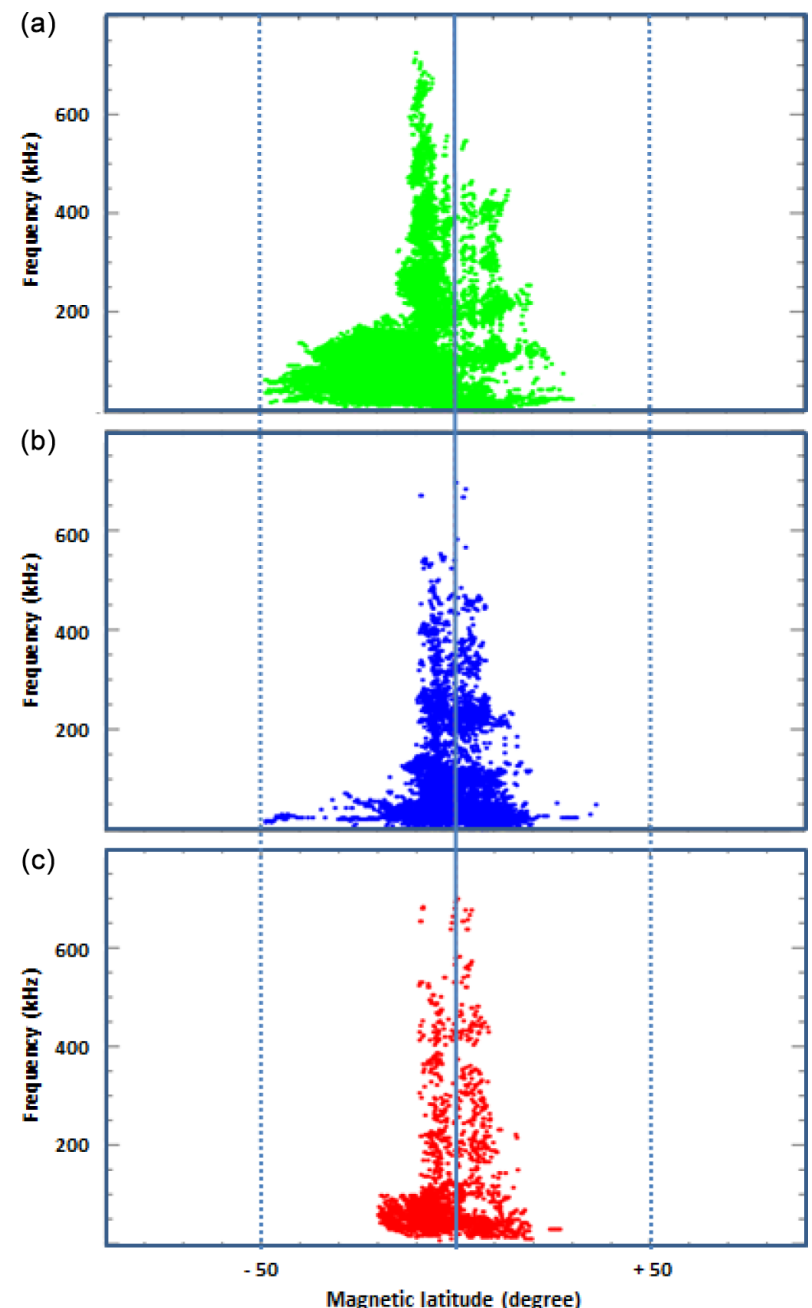

Figure 5. Variation of the power levels versus the frequency (vertical axis) and the magnetic latitude (horizontal axis) for all events. Colours are similar to those used in Fig. 4. Green, blue and red specify, respectively, three power level intervals, i.e. $10^{-3}-0.7,0.7-10$ and $10-10^{+4} \mu \mathrm{V} \mathrm{m}^{-1} \mathrm{~Hz}^{-1 / 2}$.

Also structured emissions are observed in the southern part of the magnetic equatorial plane at frequencies above $200 \mathrm{kHz}$ in magnetic latitude between -10 and $0^{\circ}$, as shown in Fig. 5a. Those structures are mainly extended in frequency, contrary to those observed in the Northern Hemisphere, which extended in magnetic latitude. We distinguish four components occurring in the following frequency bands: 200-320, 320-450, 450-570 and 570-670 kHz. At frequencies lower than $200 \mathrm{kHz}$, we note a quasi-absent structured emission in the Southern Hemisphere. Radiations continuously occur in magnetic latitude between -50 and $0^{\circ}$. In this interval, we find a positive or negative frequency drift rate of about +3.75 or $-1.25 \mathrm{kHz}$ per degree when the frequency is higher or lower than $50 \mathrm{kHz}$. The emissions are mainly confined to frequencies lower than 150 or $100 \mathrm{kHz}$ in the southern or northern part of the magnetic equatorial plane, when

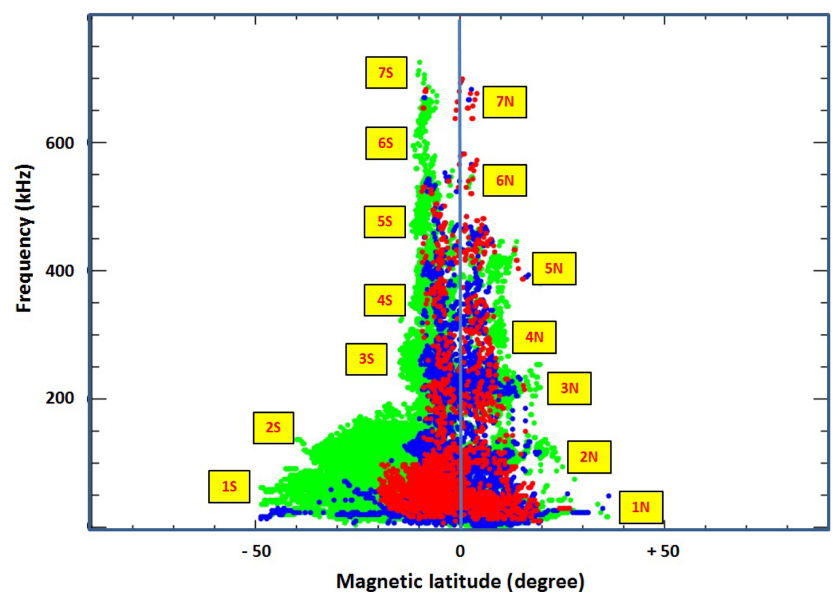

Figure 6. Overlapping of the three power levels displayed in Fig. 5. The spectral pattern is a "Christmas tree" with a "trunk" along the magnetic equatorial plane. We have indicated by numbers the main parts of the spectral pattern for the Southern Hemisphere and Northern Hemisphere. Table 1 lists the observational parameters associated with the investigated events.

the power level is between 0.7 and $10 \mu \mathrm{V} \mathrm{m}^{-1} \mathrm{~Hz}^{-1 / 2}$, as displayed in Fig. 5b. Above $150 \mathrm{kHz}$, the radiations only occur in the frequency bandwidth of 180 to about $250 \mathrm{kHz}$. The power level in the range $10-10^{4} \mu \mathrm{V} \mathrm{m}^{-1} \mathrm{~Hz}^{-1 / 2}$ is shown in Fig. 5c. The main emission is nearly symmetrical, distributed around the magnetic equatorial plane, between -10 and $+10^{\circ}$, predominantly above $100 \mathrm{kHz}$. Below this limit, the radiation covers larger magnitude latitudes from -20 to about $+20^{\circ}$.

The overlapping of the three power levels, as shown in Fig. 6, allow getting a global shape similar to a "Christmas tree" spectral pattern. We see globally that the frequencybanded emission extensively occurs at frequencies lower than $150 \mathrm{kHz}$ and starts to be less confined to the magnetic equatorial plane above this frequency limit. A cut-off appears around $50 \mathrm{kHz}$, which decreases to about a few kilohertz when approaching the magnetic equatorial plane. This cutoff is characterized by a small frequency drift rate in latitude and a power level in the interval 0.7 and $10 \mu \mathrm{V} \mathrm{m}^{-1} \mathrm{~Hz}^{-1 / 2}$, i.e. blue boundary in Fig. 6. A second cut-off can be seen when the DEMETER satellite was in the Southern Hemisphere and absent in the Northern Hemisphere. It starts at latitudes of about $-40^{\circ}$ and disappears at $-18^{\circ}$, when the frequency decreases from 150 to $50 \mathrm{kHz}$. We find that both cut-offs intersected at a frequency of about $50 \mathrm{kHz}$ when the magnitude latitude is about $-18^{\circ}$.

Table 1 lists the main observational parameters derived from Fig. 6. For each hemisphere, the opening angle of the beam, the frequency range and the magnetic latitude are indicated. Schematic representations of those beams are given, respectively, in Figs. 7 and 8 for the Southern Hemisphere and Northern Hemisphere. 
Table 1. Observational parameters of the main parts of the spectral patterns as indicated in Fig. 6.

\begin{tabular}{lcrrr}
\hline Hemisphere & Point & $\begin{array}{r}\text { Opening } \\
\text { angle }\end{array}$ & $\begin{array}{r}\text { Frequency } \\
\text { range }\end{array}$ & $\begin{array}{r}\text { Magnetic } \\
\text { latitude }\end{array}$ \\
\hline Southern & $1 \mathrm{~S}$ & $35^{\circ}$ & $30-100 \mathrm{kHz}$ & $-50^{\circ}$ to $0^{\circ}$ \\
& $2 \mathrm{~S}$ & $25^{\circ}$ & $100-200 \mathrm{kHz}$ & $-40^{\circ}$ to $0^{\circ}$ \\
& $3 \mathrm{~S}$ & $08^{\circ}$ & $200-300 \mathrm{kHz}$ & $-15^{\circ}$ to $0^{\circ}$ \\
& $4 \mathrm{~S}$ & $06^{\circ}$ & $300-450 \mathrm{kHz}$ & $-12^{\circ}$ to $0^{\circ}$ \\
& $5 \mathrm{~S}$ & $4.5^{\circ}$ & $450-550 \mathrm{kHz}$ & $-12^{\circ}$ to $-5^{\circ}$ \\
& $6 \mathrm{~S}$ & $4^{\circ}$ & $550-700 \mathrm{kHz}$ & $-12^{\circ}$ to $-6^{\circ}$ \\
& $7 \mathrm{~S}$ & $3.5^{\circ}$ & $700-730 \mathrm{kHz}$ & $-10^{\circ}$ to $-6^{\circ}$ \\
\hline \multirow{6}{*}{ Northern } & $7 \mathrm{~N}$ & $2^{\circ}$ & $630-700 \mathrm{kHz}$ & $-2^{\circ}$ to $5^{\circ}$ \\
& $6 \mathrm{~N}$ & $2^{\circ}$ & $530-580 \mathrm{kHz}$ & $-3^{\circ}$ to $5^{\circ}$ \\
& $5 \mathrm{~N}$ & $7^{\circ}$ & $350-480 \mathrm{kHz}$ & $-2^{\circ}$ to $15^{\circ}$ \\
& $4 \mathrm{~N}$ & $7^{\circ}$ & $250-350 \mathrm{kHz}$ & $0^{\circ}$ to $12^{\circ}$ \\
& $3 \mathrm{~N}$ & $12^{\circ}$ & $150-250 \mathrm{kHz}$ & $0^{\circ}$ to $20^{\circ}$ \\
& $2 \mathrm{~N}$ & $18^{\circ}$ & $70-150 \mathrm{kHz}$ & $0^{\circ}$ to $25^{\circ}$ \\
& $1 \mathrm{~N}$ & $25^{\circ}$ & $30-70 \mathrm{kHz}$ & $0^{\circ}$ to $30^{\circ}$ \\
\hline
\end{tabular}

\section{Discussion}

We discuss hereafter the frequency-banded emission as detected by the DEMETER microsatellite. First we emphasize the beaming of such emissions and how it extended and restrained around the magnetic equatorial plane. Then the similarity and the discrepancy between DEMETER frequencybanded emission and the terrestrial kilometric radiations are addressed. This is followed by a discussion on the generation mode and the source location.

\subsection{Beaming of the frequency-banded emission}

The passage of the DEMETER satellite through the magnetic equator lead to a characterization of the frequency-banded radiation recorded in the vicinity of the magnetic equatorial plane. The capability of the DEMETER satellite leads to regularly recording such a type of emissions at low altitudes around $700 \mathrm{~km}$. We have found that the radiations exhibit different spectral patterns when the frequency is smaller or bigger than $50 \mathrm{kHz}$. The satellite recorded emissions on both sides of the magnetic equator, and they appear to be more structured bands in the Northern Hemisphere. Those lasting bands indicate "stable" features in the late-evening sector at about 22 LT.

The power level distribution of the frequency-banded emission shows restrained and extended deployment around the equatorial magnetic plane. Hence the latitudinal beam is found to be about $40^{\circ}$ when the frequency is, on average, less than $100 \mathrm{kHz}$. Above this limit and up to about $800 \mathrm{kHz}$, the latitudinal beam is decreasing and found to be about $20^{\circ}$. This general picture is easily seen in Fig. 5c. However we note a clear difference in the beam when the level is less than $1 \mu \mathrm{V} \mathrm{m}^{-1} \mathrm{~Hz}^{-1 / 2}$, as shown in Fig. 5a. Hence the frequency-banded wave radiation beam is different when combing the emission recorded in the southern and northern parts of the magnetic equatorial plane. In the southern one, half of the spectral pattern is observed, i.e. beams of -25 and $10^{\circ}$, on average, in the frequency bandwidths $30-100$ and $100-800 \mathrm{kHz}$, respectively.

The beams of the frequency-banded events are found to depend on the satellite orbits with regard to the magnetic equatorial plane as shown in Figs. 7 and 8. Beams associated with the Southern Hemisphere events are observed in different frequency bandwidths. We may be deal with two source regions localized in the southern part of the magnetic equator but confined to two unlike regions with high and low plasma densities. On the other side of the equatorial magnetic plane, there are only branches or limbs. It is evident that emission diagrams are unlike that which may be due to the combined effects of multiple beams associated with sources localized in different regions. Beams in Figs. 7 and 8 may be considered an overlapping of single beams. Each one can be associated with one narrow-band structure as shown in Figs. 1 and 2.

Figure 9 displays the variation of the $L$ shell associated with the frequency-banded events versus the magnetic latitude of the satellite. The power level is principally found to increase in the $L$-shell range between 1 and 1.4 when the magnetic latitude of DEMETER is in between -20 and $+20^{\circ}$. Those orbital parameters are related to the beam radiated by the source emission which crossed DEMETER trajectories.

\subsection{Similarity and discrepancy with the terrestrial kilometric emission}

Frequency-banded emission features, as investigated in this paper, allow us to address questions concerning its origin. We have found some spectral patterns which are similar to those reported in the literature in the case of the terrestrial kilometric emissions.

First, we have described changes of the spectral emissions at frequencies of about $50 \mathrm{kHz}$. Such frequencies boundaries are similar to those observed by other satellite observations, like Cluster, Geotail and IMAGE. Hence the terrestrial kilometric radiation is trapped and escaping when the frequency is, respectively, smaller and bigger than $50 \mathrm{kHz}$. The spectral features are often comparable, and the main alternations may be due to the instrumental time, frequency resolutions and also the satellite orbits with regard to the source locations. Hence Green and Boardsen (2006) show a typical sample of the kilometric continuum recorded by the RPI experiment on board IMAGE during the passage of the magnetic equatorial plane. In their Fig. 2, one can observe the presence of parallel narrow bands at frequencies above $30 \mathrm{kHz}$. Such narrow bands have a morphological similarity with those displayed in Fig. 6 of our paper. The AKR-X (Analyzer of Kilometric Radio emissions eXperiment) experiment on board INTERBALL-1 provided similar emissions particularly in the Southern Hemisphere at low magnetic latitudes with an 


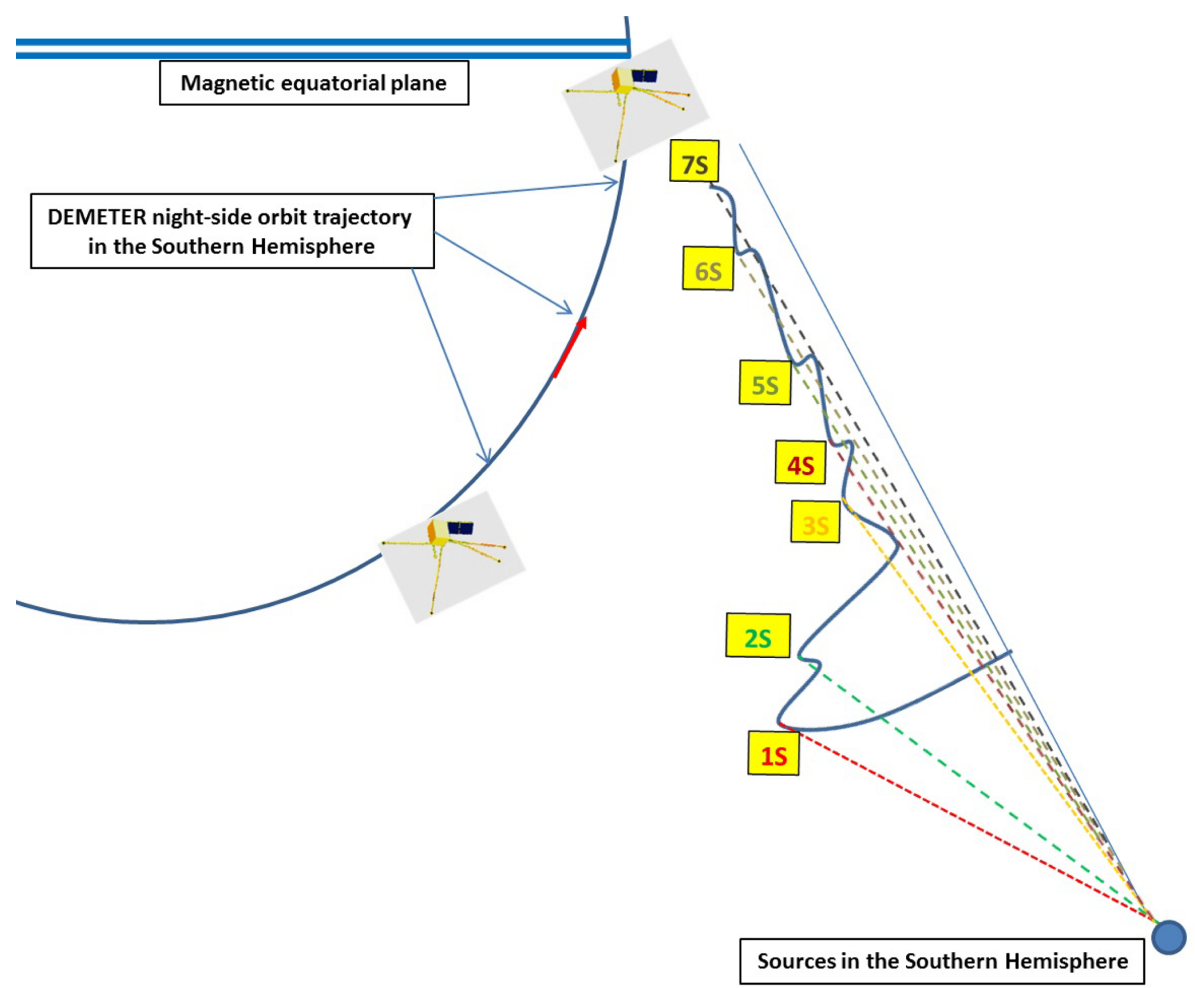

Figure 7. Sketch of the beams observed in the Southern Hemisphere for specific magnetic latitudes and frequencies (i.e. $1 \mathrm{~S}, 2 \mathrm{~S}, 3 \mathrm{~S}, 4 \mathrm{~S}, 5 \mathrm{~S}$, $6 \mathrm{~S}$ and $7 \mathrm{~S}$ ) as listed in Table 1.

$L$-shell value of about 1.2, as reported by Kuril'chik et al. (2001, 2007). Observations at fixed frequencies $(100,252$, 500 and $749 \mathrm{kHz}$ ) allowed for the analysis of the spectral character of such emissions. Authors showed that the terrestrial kilometric radiation occurrence depends on the solar activity. Such radiation is regularly recorded during quiet solar activity. Our observations were registered in the begging of the year 2010, nearly 18 months after the minimum of solar activity, i.e. August 2008. Also the spectral pattern looks like a Christmas tree, as also reported by Green and Boardsen (2006) in their review of the kilometric continuum radiation, and it is confined to the magnetic equatorial plane.

Despite those common spectral features, several other observational aspects are different when combining the terrestrial kilometric radiation and the frequency-banded wave emission. The investigated DEMETER emission is detected at distance of about $1.1 R_{\mathrm{E}}$, which is generally not the case of the terrestrial kilometric emission. For instance, Geotail and Cluster observations recorded radiation at more than $15 R_{\mathrm{E}}$, as reported by Hashimoto et al. (1999) and Décréau et al. (2004), respectively. Also, the trapped or the escaping component is linked to terrestrial kilometric radiation recorded, respectively, between the plasmasphere and the magnetosphere or outside of the magnetosphere. This radiation propagate largely in the free space in the LO (left-hand polarized ordinary) mode above the local plasma frequency linked to sources at or very near the plasmapause (Hashimoto et al., 2006). Also the gyro-frequency is found to be smaller than the trapped and the escaping frequencies, as recorded by the RPI experiment on board IMAGE (see Fig. 2 of Green and Boardsen, 2006). All those observational parameters are not similar to those reported in the case of the frequency-banded wave emission recorded by DEMETER satellite.

\subsection{Micro-scale features of the inner part of the plasmasphere}

It is clear that both radiations have common spectral features but several discrepancy observational aspects linked to the generation mechanism. However the source locations should be the plasmasphere. Hence the terrestrial kilometric radiation is linked to plasmaspheric sources with emission beams oriented towards the magnetosphere. Figures 7 and 8 showed the emission beams which interact with DEMETER orbits. The sources are localized poleward of the plasmasphere. This means that the DEMETER orbits cross the plasmaspheric hollow cones for a few dozen kilometres. Probably such restricted regions may be associated with the $Z$-mode waves, which are linked to the free escaping LO mode as suggested by Jones (1976) in his model. In such a region, the $Z$-mode waves are considered to be trapped and later converted into the LO mode associated with the terrestrial kilometric radiation. Later on, Goertz and Strangeway (1995) derived from 


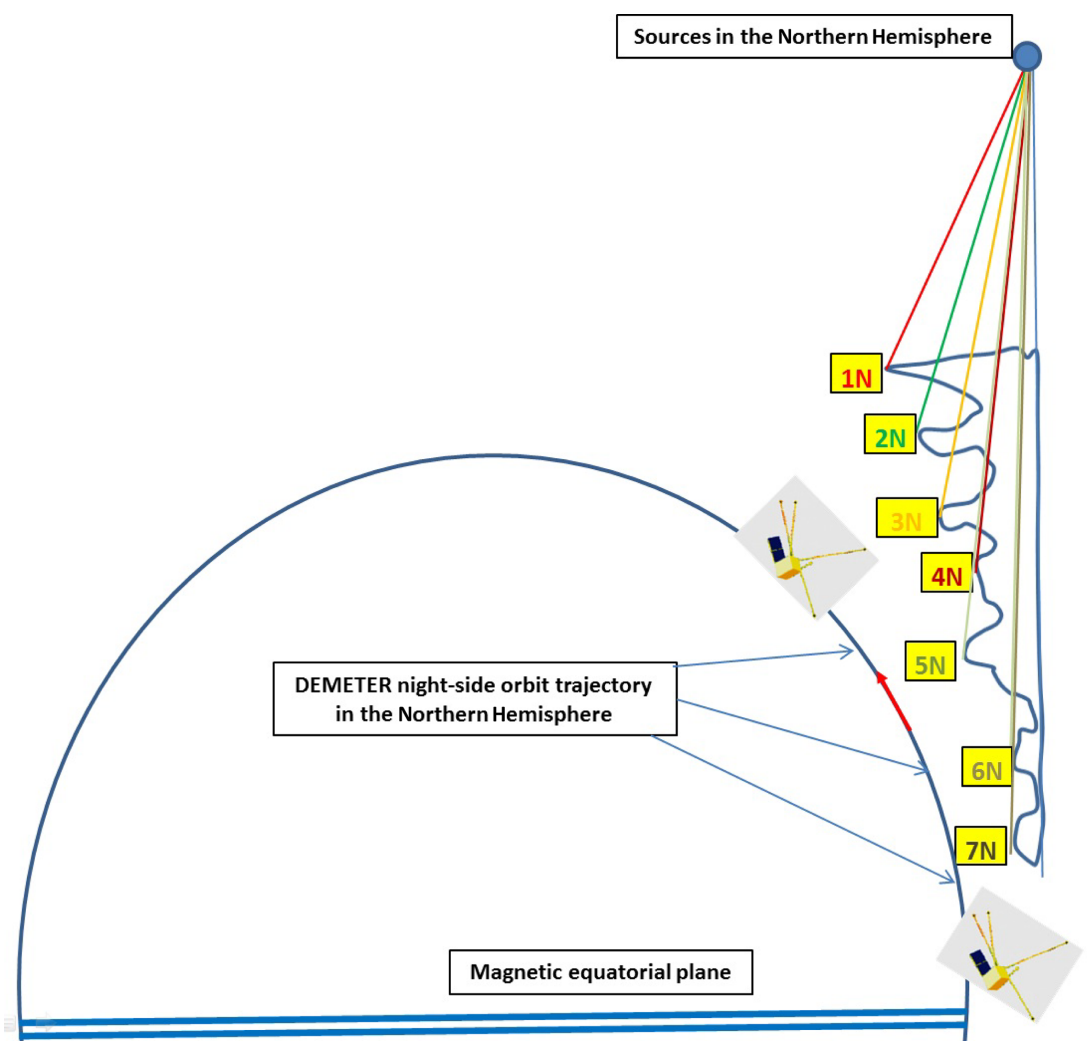

Figure 8. Sketch of the beams observed in the Northern Hemisphere for specific magnetic latitudes and frequencies (i.e. $1 \mathrm{~N}$, $2 \mathrm{~N}$, $3 \mathrm{~N}$, $4 \mathrm{~N}$, $5 \mathrm{~N}, 6 \mathrm{~N}$ and $7 \mathrm{~N}$ ) as listed in Table 1.

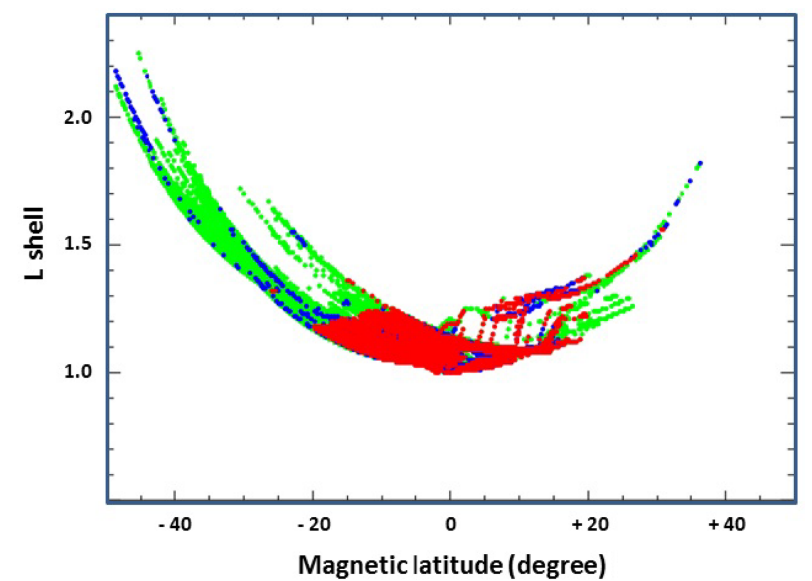

Figure 9. Variation of the frequency-banded wave emission versus the $L$ shell and the magnetic latitude.

the Appleton-Hartree dispersion relation the whistler wave propagation in the case when the electron plasma frequency is smaller than the gyro-frequency. Carpenter et al. (2003) found a similar region where ray paths of $Z$-mode echoes from radio sounding were recorded by the IMAGE satellite in the polar regions. Also Sonwalkar et al. (2004) investigated the whistler mode echoes from radio sounding and found $f_{\mathrm{p}}$ to be smaller than $f_{\mathrm{g}}$ in the region poleward of the plasmapause. Green and Boardsen (2006) investigated and reported on the linear-mode-conversion theory based on the model of Jones. Authors showed profiles of plasmaspheric plasma frequency, taking into consideration the $Z$-mode frequency and the equatorial gyro-frequency. Regions of a sharp plasma gradient are found and shown in Fig. 5 of their paper.

We estimate the relationship between the $Z$-mode frequency $\left(f_{\mathrm{z}}\right)$, the plasma frequency $\left(f_{\mathrm{p}}\right)$ and the gyro-frequency $\left(f_{\mathrm{g}}\right)$ using the following formula: $f_{\mathrm{z}}=$ $\left(f_{\mathrm{g}} / 2\right)\left[-1+\left(1+4\left(f_{\mathrm{p}} / f_{\mathrm{g}}\right)^{2}\right)^{1 / 2}\right]$ (Carpenter et al., 2003). Figure 10 displays the variation of the three frequencies (i.e. $f_{\mathrm{z}}, f_{\mathrm{p}}$ and $f_{\mathrm{g}}$ ) versus the geocentric distance. The trapping region is localized between the lower and the higher $f_{\mathrm{Z}}$ (green in Fig. 10), mainly between 1 and $100 \mathrm{kHz}$ and extended up to $700 \mathrm{kHz}$. The plasma frequency follows the trapping region, starting at about 10 and going up to $800 \mathrm{kHz}$. The gyro-frequency appears at higher frequencies, i.e. above $800 \mathrm{kHz}$. Those features are comparable to previous investigations, e.g. Gurnett et al. (1983) and Carpenter et al. (2003), in polar regions.

It is important to note that the frequency-banded emissions may be due to the interaction and/or the co-existence of electrostatic and whistler waves. Hence Bell and Ngo (1990) considered theoretically the generation, in the case of the density 


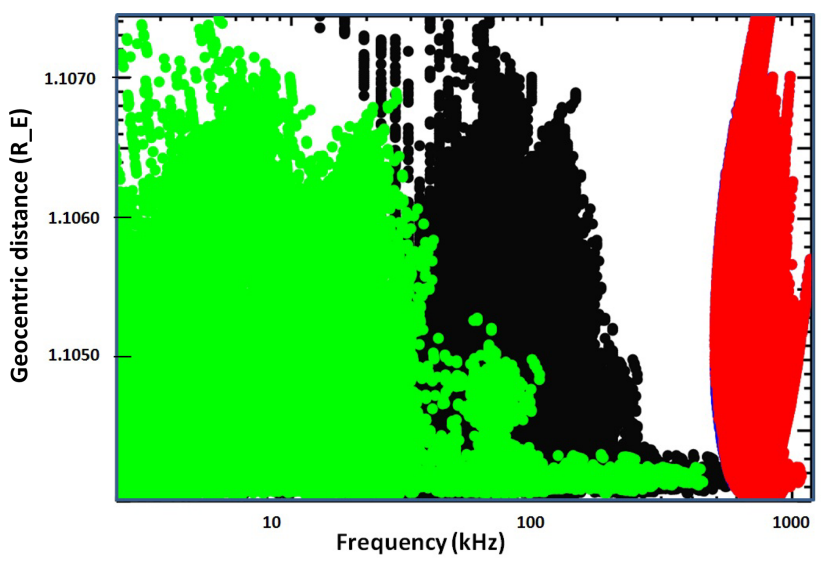

Figure 10. Variations of the frequency-banded emission versus the geocentric distance expressed in $R_{\mathrm{E}}$. The green, black and red are associated, respectively, with $f_{\mathrm{Z}}, f_{\mathrm{p}}$ and $f_{\mathrm{g}}$ frequencies.

cavity or gradient, of whistler waves by lower-hybrid waves. Such a co-existence of both waves has been investigated by An et al. (2017) using Darwin particle-in-cell (PIC) simulation. Such models have been recently invoked by Vartanyan et al. (2016) and Li et al. (2017) in the investigation, respectively, of the generation of whistler waves and chorus wave modulation observed on board DEMETER and the Van Allen Probes.

\section{Conclusion}

We have investigated the frequency-banded wave radiation recorded by the ICE experiment on board DEMETER. DEMETER orbits allow us to regularly record this radiation where, in the optimal case, about 13 events are daily registered. The power level is found in the interval between $10^{-3}$ and $10^{+4} \mu \mathrm{V} \mathrm{m}^{-1} \mathrm{~Hz}^{-1 / 2}$. The spectral analysis leads to finding a "tree spectral shape", which is the traces of the beaming of the frequency-banded wave radiation. We have shown that those beams are not similar and depend on the emission frequency and the magnetic latitude. The DEMETER frequency-banded emission can be comparable to the wellknow terrestrial kilometric radiation. However several other observational aspects are different when combining both emissions, in particular the generation modes. We suggest that the DEMETER frequency-banded emissions are linked to a $Z$-mode micro-scale region. This trapping $Z$-mode region can only be detected between Earth's ionosphere and the plasmasphere. The hollow cones of these frequency-banded wave emissions are crossed by the DEMETER orbits at altitudes lower than $700 \mathrm{~km}$. Probably the source regions of the DEMETER frequency-banded emission should be the plasmasphere, like the terrestrial kilometric radiation. IMAGE investigations have reported about the time evolution of the plasmasphere in particular on the pre-midnight sector
(Sandel et al., 2003) and the presence of density structures (Darrouzet et al., 2009) at smaller scales. We may consider that DEMETER orbits allow for investigating the inner part of the plasmasphere when other missions (i.e. Geotail, IMAGE and INTERBALL) lead to studying the outer part of the plasmasphere.

Data availability. Instrument Champ Électrique (ICE) data used in this study are available from the DEMETER Data Server (http:// demeter.cnrs-orleans.fr/, last access: 13 May 2019) and from http: //www.cdpp.eu (last access: 19 June 2020). For further information please contact the corresponding author.

Author contributions. MYB carried out the analysis and wrote the paper. PHMG, VD and HL helped with the interpretation of the data. All authors contributed to the discussion.

Competing interests. The authors declare that they have no conflict of interest.

Acknowledgements. Authors thank the Centre National d'Etudes Spatiales (CNES) and Jean-Jacques Berthelier (PI of the ICE instrument) for the use of the data. The authors are grateful to the anonymous reviewers, whose comments helped them to improve the article.

Financial support. This research has been supported by the Austrian Academy of Sciences and the Russian Academy of Sciences.

Review statement. This paper was edited by Yoshizumi Miyoshi and reviewed by two anonymous referees.

\section{References}

An, X., Bortnik, J., Van Compernolle, B., Decyk, V., and Thorne, R.: Electrostatic and whistler instabilities excited by an electron beam, Phys. Plasmas, 24, 072116, https://doi.org/10.1063/1.4986511, 2017.

Bell, T. F. and Ngo, H. D.: Electrostatic lower hybrid waves excited by electromagnetic whistler mode waves scattering from planar magnetic-field-aligned plasma density irregularities, J. Geophys. Res., 95, 149-172, 1990.

Berthelier, J. J., Godefroy, M., Leblanc, F., Malingre, M., Menvielle, M., Lagoutte, D., Brochot, J. Y., Colin, F., Elie, F., Legendre, C., Zamora, P., Benoist, D., Chapuis, Y., Artru, J., and Pfaff, R.: ICE, the electric field experiment on DEMETER, Planet. Space Sci., 54, 456-471, 2006.

Boudjada, M. Y., Biagi, P. F., Al-Haddad, E., Galopeau, P. H. M., Besser, B., Wolbang, D., Prattes, G., Eichelberger, H., Stangl, G., Parrot, M., and Schwingenschuh, K.: Reception conditions 
of low frequency (LF) transmitter signals onboard DEMETER micro-satellite, Phys. Chem. Earth, 102, 70-79, 2017.

Brown, L. W.: The galactic radio spectrum between $130 \mathrm{kHz}$ and $2600 \mathrm{kHz}$, Astrophys. J., 180, 359-370, 1973.

Burch, J. L.: IMAGE mission overview, Space Sci. Rev., 91, 1-14, 2000.

Carpenter, D. L., Bell, T. F., Inan, U. S., Benson, R. F., Sonwalkar, V. S., Reinisch, B. W., and Gallagher, D. L.: Z-mode sounding within propagation "cavities" and other inner magnetospheric regions by the RPI instrument on the IMAGE satellite, J. Geophys. Res., 108, 1421, https://doi.org/10.1029/2003JA010025, 2003.

Darrouzet, F., Gallagher, D. L., André, N., Carpenter, D. L., Dandouras, I., Décréau, P. M. E., De Keyser, J., Denton, R. E., Foster, J. C., Goldstein, J., Moldwin, M. B., Reinisch, B. W., Sandel, B. R., and Tu, J.: Plasmaspheric Density Structures and Dynamics: Properties Observed by the CLUSTER and IMAGE Missions, Space Sci. Rev., 145, 55-106, 2009.

Décréau, P. M. E., Ducoin, C., Le Rouzic, G., Randriamboarison, O., Rauch, J.-L., Trotignon, J.-G., Vallières, X., Canu, P., Darrouzet, F., Gough, M. P., Buckley, A. M., and Carozzi, T. D.: Observation of continuum radiations from the Cluster fleet: first results from direction finding, Ann. Geophys., 22, 2607-2624, https://doi.org/10.5194/angeo-22-2607-2004, 2004.

Décréau, P. M. E., Fergeau, P., Krasnoselskikh, V., Le Guirriec, E., Lévêque, M., Martin, Ph., Randriamboarison, O., Rauch, J. L., Sené, F. X., Séran, H. C., Trotignon, J. G., Canu, P., Cornilleau, N., de Féraudy, H., Alleyne, H., Yearby, K., Mëgensen, P. B., Gustafsson, G., André, M., Gurnett, D. C., Darrouzet, F., Lemaire, J., Harvey, C. C., Travnicek, P., and Whisper experimenters (Table 1): Early results from the Whisper instrument on Cluster: an overview, Ann. Geophys., 19, 1241-1258, https://doi.org/10.5194/angeo-19-1241-2001, 2001.

El-Lemdani Mazouz, F., Rauch, J. L., Décréau, P. M. E., Trotignon, J. G., Vallières, X., Darrouzet, F., Canu, P., and Suraud, X.: Wave emissions at half electron gyroharmonics in the equatorial plasmasphere region: CLUSTER observations and statistics, Adv. Space Res., 43, 253-264, 2009.

Goertz, C. K. and Strangeway, R. J.: Plasma waves, in: Introduction to Space Physics, edited by: Kivelson, M. G. and Russell, C. T., p. 356, Cambridge Univ. Press, New York, 1995.

Green, J. L. and Boardsen, S. A.: Kilometric continuum radiation, Radio Sci. Bull., 318, 34-41, 2006.

Grimald, S. and Santolik, O.: Possible wave modes of wideband nonthermal continuum radiation in its source region, J. Geophys. Res., 115, A06209, https://doi.org/10.1029/2009JA014997, 2010 .

Grimald, S., Décréau, P. M. E., Canu, P., Rochel, A., and Vallières, X.: Medium latitude sources of plasmaspheric non thermal continuum radiations observed close to harmonics of the electron gyrofrequency, J. Geophys. Res., 113, A11217, https://doi.org/10.1029/2008JA013290, 2008.

Grimald, S., El-Lemdani Mazouz, F., Foullon, C., Décréau, P. M. E., Boardsen, S. A., and Vallières, X.: Study of non-thermal continuum patches: wave propagation and plasmapause study, J. Geophys. Res., 116, A07219, https://doi.org/10.1029/2011JA016476, 2011.

Gurnett, D. A.: The Earth as a radio source: The nonthermal continuum, J. Geophys. Res., 80, 2751-2763, 1975.
Gurnett, D. A. and Shaw, R. A.: Electromagnetic radiation trapped in the magnetosphere above the plasma frequency, J. Geophys. Res., 78, 8136-8148, 1973.

Gurnett, D. A., Shawhan, S. D., and Shaw, R. R.: Auroral hiss, $\mathrm{Z}$-mode radiation, and auroral kilometric radiation in the polar magnetosphere: DE 1 observations, J. Geophys. Res., 88, 329340, https://doi.org/10.1029/JA088iA01p00329, 1983.

Hashimoto, K., Calvert, W., and Matsumoto, H.: Kilometric continuum detected by GEOTAIL, J. Geophys. Res., 104, 28645 28656, 1999.

Hashimoto, K., Green, J. L., Anderson, R. R., and Matsumoto, H.: Review of kilometric continuum, in: Geospace Electromagnetic Waves and Radiation, Lect. Not. in Phys., edited by: LaBelle, J. W. and Treumann, R. A., Springer, New York, 687, 37-54, 2006.

Jones, D.: Source of terrestrial nonthermal radiation, Nature, 260, 686-689, 1976.

Kuril'chik, V. N., Boudjada, M. Y., and Rucker, H. O.: INTERBALL-1 observations of the plasmaspheric emissions related to terrestrial "continuum" radio emissions, in: Planetary Radio Emissions V, edited by: Rucker, H. O., Kaiser, M. L., and Leblanc, Y., Austrian Academy of Sciences Press, Vienna, 325335, 2001.

Kuril'chik, V. N., Boudjada, M. Y., Rucker, H. O., and Kopaeva, I. F.: Observations of Electromagnetic Emissions inside the Earth's Plasmasphere from the INTERBALL-1 Satellite, Cosmic Res., 45, 455-460, 2007.

Kurth, W. S., Gurnett, D. A., and Anderson, R. R.: Escaping nonthermal continuum radiation, J. Geophys. Res., 86, 5519-5531, 1981.

Li, J., Bortnik, J., An, X., Li, W., Thorne, R. M., Zhou, M., Kurth, W. S., Hospodarsky, G. B., Funsten, H. O., and Spence, H. E.: Chorus Wave Modulation of Langmuir Waves in the Radiation Belts, Geophys. Res. Lett., 44, 11713-11721, 2017.

Parrot, M. and Berthelier, J.-J.: AKR-like emissions observed at low altitude by the DEMETER satellite, J. Geophys. Res., 117, A10314, https://doi.org/10.1029/2012JA017937, 2012.

Parrot, M., Inan, U. S., Lehtinen, N. G., and Pincon, J. L.: Penetration of lightning MF signals to the upper ionosphere over VLF ground-based transmitters, J. Geophys. Res., 114, A12318, https://doi.org/10.1029/2009JA014598, 2009.

Sandel, B. R., Goldstein, J., Gallagher, D. L., and Spasojevic, M.: Extreme ultraviolet imager observations of the structure and dynamics of the plasmasphere, Space Sci. Rev., 109, 25-46, 2003.

Sonwalkar, V. S., Carpenter, D. L., Bell, T. F., Spasojevic, M., Inan, U. S., Li, J., Chen, X., Venkatasubramanian, A., Harikumar, J., Benson, R. F., Taylor, W. W. L., and Reinisch, B. W.: Diagnostics of magnetospheric electron density and irregularities at altitudes $<5000 \mathrm{~km}$ using whistler and $\mathrm{Z}$ mode echoes from radio sounding on the IMAGE satellite, J. Geophys. Res., 109, A11212, https://doi.org/10.1029/2004JA010471, 2004.

Vartanyan, A., Milikh, G. M., Eliasson, B., Najmi, A. C., Parrot, M., and Papadopoulos, K.: Generation of whistler waves by continuous HF heating of the upper ionosphere, Radio Sci., 51, 11881198, 2016. 